\title{
Der Schauplatz der Passion des römischen Bischofs Sixtus II.
}

\author{
Von P. Corssen in Berlin-Dahlem.
}

Xistum autem in coemeterio animadversum sciatis VIII. Id. Aug. die et cum eo diacones quattuor, schreibt Cyprian an seinen Amtsgenossen Successus ep. LXXX 1.

So einfach diese Nachricht klingt, so schwer verständlich ist sie. Denn die Benutzung einer christlichen Begräbnisstätte als Richtplatz durch die römische Behörde läßt sich mit unserer Kenntnis des römischen Strafverfahrens schwer vereinigen.

Im Jahre 257 hatten die Kaiser das Betreten der Friedhöfe bei Todesstrafe verboten, ${ }^{1}$ und das kaiserliche Reskript vom Jahre 258 begann nach Cyprians Angabe in dem ebengenannten Briefe mit der Bestimmung ut episcopi et presbyteri et diacones in continenti animadvertantur. War Sixtus auf dem Friedhof überrascht und dort auf der Stelle hingerichtet worden? Auf ein solches Verfahren hätte kaum der Ausdruck animadverti angewendet werden dürfen. Denn dieser setzt ein richterliches Mandat voraus, und nie ist über die Christen einfach eine Proskription verhängt worden, auch nicht in der valerianischen Verfolgung. Daß auch in ihr die gerichtlichen Formen beobachtet wurden, beweist u. a. der Prozeß Cyprians. Cyprian gibt daher schwerlich den Wortlaut des Ediktes wieder; jedenfalls ist in continenti nur in bezug auf eine vorhergègangene Gerichtsverhandlung zu verstehen. Die Bestimmung ging nicht dahin, daß die gerichtliche Verhandlung gegen die Christen aufgehoben wurde, sondern daß die Strafvollziehung unmittelbar nach der Sentenz erfolgen sollte, wie ja auch mit Cyprian verfahren wurde, was zwar Regel, aber keineswegs eine allgemein gültige Bestimmung war. ${ }^{2}$ Cyprian, so scheint es dem-

1 Acta Cypriani I: praeceperunt etiam, ne in aliquibus locis conciliabula faciant nec coemeteria ingrediantur; si quis itaque hoc tam salubre praeceptum non observaverit, capile plectetur.

2 Mommsen, Römisches Strafrecht, S. 911. 
nnch, hat gemeint, das von dem Richter luber Sixtus gefallte Urteil sei auf dem Friedhof vollstreckt worden. So hat auch De Rossi den Hergang dargestellt.

Allcin auch diesc Annahme hat sehr gewichtige Bedenken gegen sich. Man könnte zwar eine Erklärung in der Bemerkung Mommsens zu finclen glauben, daß es in der Spät/cit lublich gewesen sei, den Verbrecher an dem Tatort zum abschreckenden Beispiel hinzurichten.' Aber dies Verfahren war auf Straßenräuber beschränkt. ${ }^{2}$ Wenn es aber auf Sixtus angewendet worden wäre, so hätte es den Zweck der Abschreckung nur sehr unvollkommen erreicht, da ja das Coemeterium durchaus nicht den Charakter der Öffentlichkeit hatte und die Christen zur Anwesenheit bei der Exekution erst hätten gepreßst werden müssen. Aul3erdem aber würde man in dem Coemeterium schwerlich den Tatort des Verbrechens erkennen können, wegen dessen Sixtus zum Tode verurteilt wurde, sclbst wenn cr darauf betroffen war. Denn die Verurteilung hatte nicht nach dem Edikt des Jahres 257 , sondern nach dem des Jahres $258 \mathrm{zu}$ erfolgen, und nach diesem genugte zur Verurteilung bei den Geistlichen offenbar die bloße Feststellung ihres Charakters, wobei abcr gewiß ein Widerruf von seiten des Angeklagten zu seiner Freisprechung führte, auch wenn er eine Versammlung auf einem Friedhof abgchalten hatte.

Ganz anders als bei Cyprian stellt sich die Hinrichtung des Sixtus in der römischen Tradition dar.

Die Akten des Sixtus ${ }^{3}$ werden zwar selbst von den Bollandisten für interpoliert crklärt, und ihre spätere Abfassung verrät sich an ihnen gleich am Anfang nur allzu deutlich in dem Satze: Decius Caesar et Valerianus praefectus iusserunt. Immerhin sind die Akten älter als Ambrosius, der sie in derselben Form gelesen haben muß wie wir. Denn er zitiert daraus ein großes Stück in wörtlicher Übereinstimmung mit dem Text der Bollandisten.4 Demgegenüber will die kleine Abweichung nicht viel verschlagen, auf welche die Bollandisten die Behauptung gründen, daB ihm die Akten in einer älteren Gestalt vorgclegen haben. Nach Ambrosius redet nämlich Laurentius den Bischof in dem Augenblick an, als er zum Martyrium geführt wurde, während

1 S. 914.

2 Dig. XXXXVIII 19, 28, 15. Famosos latrones in his locis, ubi grassali sunt, furca figeniles compluribus placuit, ut et conspectu deterreantur alii ab isdem facinoribus et solacio sit cognatis et adfinibus interemplorum codem loco poena readila, in quo latrones homicitia fecissent.

3 Acta Sunctorum Aug. 6. 4 Ambrosius, De officiis I 41 § 204. 
es nach der Akton geschah, als Sixtus nach der ersten Verhandlung ins Gefängnis zurockgefahrt wurde. Hier liegt nichts anderes als cine Ungenauigkeit des Ambrosius vor, wenn man es aberhaupt so nennen will. Denn auf die Frage des Laurentius Quo progrederis' ist dic Antwort Ad martyrium dem Sinn der Akten ganz gemät. Dati zwisichen der Zeit der Prage und der Hinrichtung ein Aufenthalt in Gefängnis und eine zweite Verhandlung liegt, ändert an dem Endresultat nichts, ganz abgesehen davon, dab man doch das Gefängnis auch zu dem Martyrium rechnen muß.

So verschwommen aber auch die historischen Vorstellungen in den Akten sind, so deutlich beweisen ihre zuverlässigen topographischen Angaben ihren römischen Ursprung. Bïinen besonders guten Findruck macht der Abschnitt uber die eigentlicho Passion, der folgendermalen lautet:

c. 8. Sanctum vero Syxtum cpiscopum at ficlicissimum et Aga. fritum diaconcs duxcrunt in clivum Martis ante templum at ibidcm decollatus est cum duobus diaconibus et dimiscrunt corfora cortum in pla. tea octavo Jd.Aug. Noctu etiam venerunt clevici at preshytcri st dincomi at maxima pars Cluristianurum at collegerunt corpora sanclonum at sepelicrunt b. Syxtum opiscojum at martyrem in crypla in cocmeterio Calisti in cadem via, sanctos vero Vielicissimum et Agrafitum marlyres at diacones sepolierunt in coconedcrio Praclextnti sub die VIJIo Jd. Aug.

Das Datum stimmt genau mit Cyprians Angabe Ubercin, ihre Unabhangeigkeit aber beweisen die Akten durch die Verschiedenheit in der Angabe der kichtstátte und der Zahl der Opier, sowic durch die Hinzufogung des Eegrähnisplatzes aller drei Märtyrer. Die Kichtigkeit der letzten Angabe ist durch die Ausgrabungen bestitigt worden, ' und hegen die erste ist an und fur sich nichts einzuwenden.

Auf das Martyrium des Sixtus hat De Rossi ${ }^{2}$ eine Inschrift des Darnasus bezogen, die gewöhnlich auf Stephanus, den Vorgänger des Sixtus, gedeutet wird, und so eine Úbercinstimmung zwischen Damasus und Cyprian zu konstatieren versucht. Dieser Versuch ruft sofort prinzipielle Bedenken hervor, denn es wäre sehr merkwlirdig, wenn Damasus sich mit der römischen Tradition in Widerspruch gesct/zt haben sollte. Welches Ansehen abur dic Akten des Sixtus zu Damasus' Zeit uberhaupt hatten, beweist der Gebrauch, den Ambrosius von ihner semacht hat. Sobald man aber daran keht, die Verse sclbst

' Ie Kusss, Juma Sotter ranea II 98f. und Armellin, Gll antichl cimsteri eristiunt, p. Sur. Lanelbat S. 26 ff. 
vorurteilsfrei $z u$ interpretieren, luberzeugt man sich, daß die alte Erklärung zu Recht besteht.

Tempore quo gladius secuit pia viscera matris

Hic positus rector caelestia izssa docebam.

Adveniunt subito, rapiunt qui forte sedentem.

Militibus missis populi tunc colla dedere.

Mox sibi cognovit senior quis tollere vellet

Palmam, seque stumque caput prior obtulit ipse

Impatiens, feritas posset ne laedere quemquam.

Ostendit Christus, reddit qui praentia vitae,

Pastoris meritum, numerum gregis ipse tuetur.

Auch De Rossi hält es für ausgeschlossen, daß Sixtus ohne Verbör hingerichtet sei. Er deutet daher die Worte rapiunt qui forte sedentem in dem Sinne, daß der Bischof vor den Richter geschleppt und dann alsbald nach der Urteilsverkündung an den Ort seines Verbrechens zurückgeführt und dort zum abschreckenden Beispiel für die Christen enthauptet worden sei. Damit wird in die Verse etwas hineingetragen, wovon auch Cyprian nicht das geringste sagt.

Die Verse nennen den Namen des Märtyrers nicht und deuten ihn auch nicht irgendwie an. Nur darüber lassen sie keinen $Z$ weifel, daß er an der Spitze der Gemeinde stand. Die Laien bieten ihren Hals für ihren Bischof dar, aber dieser sieht darin ein Spiel des Teufels. Er will sich die Krone nicht rauben lassen und kommt ihnen zuvor, damit die wilden Schergen niemand verletzen. So verschafft er sich das Martyrium und schirmt die Gemeinde. Außer dem Bischof kommt niemand um. Schon aus diesem Grunde kann man nicht an Sixtus denken, mit dem nach seinen Akten und dem Grabbefunde zwei, nach Cyprian vier, nach dem Liber pontificalis sechs Diakonen starben. Damasus bewegt sich in Andeutungen, die kein-vollständiges Bild ergeben. Der Vorgang ist, wie er ihn schildert, nur denkbar, wenn man annimmt, daß sich bei dem Eintreten der Polizisten ein allgemeiner Tumult erhob, in welchem der Bischof diese auf sich zog und sie so reizte, daß sie ihn erschlugen. Eine dem völlig entsprechende Darstellung geben die Akten des Stephanus nicht, aber in wesentlichen Stücken stimmen sie mit Damasus überein. Nach ihnen war Stephanus von Valerian zum Tode verurteilt für den Fall, daß er sich weigern würde, den Mars in seinem Tempel auf dem clivus Martis anzubeten. Der Tempel wird aber durch ein Wunder zerstört, Stephanus entkommt den Häschern und begibt sich mit allen Christen nach dem Coemeterium der h. Lucina. Hier wird er von den Soldaten, 
die Valerianus ausgesendet hatte, bei dem Darbringen der Hostie betroffen und auf seinem Stuhle enthauptet: Missi sunt plurimi prioribus militum ... codem loco sic in sua decollatus est sede. Daran klingen die Verse des Damasus an: Adveniunt subito rapiunt qui forte sedentem. Militibus missis . . caput obtulit ipse. Aber freilich steht in den Akten nichts davon, daß die Gemeinde sich für ihren Bischof opfern wollte. Die Hinrichtung ist nach ihnen durch das voraufgegangene Urteil gerechtfertigt. Der Leib des Stephanus wird an Ort und Stelle in der später mit dem Coemeteriun des Calixtus vereinigten Krypta der Lucina beigesetzt: Cuius corpus sepeliernent in eadem crypta cum ipsa sede, ubi adstans sanguis eius effusus est, in loco quiti appellatur hodie coeneterium Callisti.

De Rossi hat diese Worte so verstanden, als wenn Stephanus in der Papstgruft beigesetzt sei (II 80). Allein wenn hervorgehoben wird, $\mathrm{da} ß$ der Ort "heute" cocmeterium Callisti heiße, so hieß er eben früher anders, und da vorher erzählt worden war, daß Stephanus sich auf den Friedhof der Lucina begeben hatte: Stephanus pergit cum omnibus Christianis ad coemeterium sanctae Lucinae, er aber diesen nicht wieder verlassen hatte, so ist gemeint, daß er an Ort und Stelle neben dem Stuhl, an dem er sein Blut verspritzt hatte, begraben worden war. Es wird also durch diese Angabe bezeugt, was durch das Ergebnis der Ausgrabungen bestätigt ist, ${ }^{1}$ daß der Friedhof der Lucina ursprünglich von dem des Calixtus getrennt war und erst später mit ihm vereinigt ist. Ein anderes Zeugnis dafür hat De Rossi I 276 selbst aus dem Papstbuch in dem Leben des Papstes Cornelius angeführt: cuius (Corneli) corpus noctu collegit beata Lucina cum clericis et sepelivit in crypta iuxta cymiterium Calisti in Appia in praedio suo. Auch einige Handschriften der Akten des Cornelius, aus denen das Papstbuch diese Notiz geschöpft hat, haben iuxta coemeterium statt in coemeterio.

Aber für De Rossi handelt es sich ganz besonders um den Nachweis, daß die Inschrift Tempore quo gladius für die Papstgruft angefertigt und in der Papstgruft angebracht war. Dafür hat er II $25 \mathrm{f}$. ein ganz besonders bestechendes Argument geltend gemacht.

In der Papstgruft fand er winzige Reste von drei Zeilen auf zwei Stücken einer Tafel, in denen er Fragmente der drei letzten Zeilen eben der Inschrift Tempore zu erkennen glaubte. Auf dem ersten Stück las er untereinander ED und IP, auf dem zweiten REG, was sich in die drei letzten Zeilen in dieser Weise einfügte:

1 Roma sott. I Anhang S. $69 \mathrm{ff}$. 
Impatiens feritas posset ne laEDere quemquam Ostendit Christus, reddit quI Pracmia vitae, Pastoris meritum, mumerum $g R E G$ is ipse tuetur.

Niemand wird in dieser Kombination die Genialität des großen Gelehrten verkennen. Allein in der Abbildung, die De Rossi von den Fragmenten gibt, sind mit absoluter Sicherheit nur die Buchstaben $\mathbf{E}$ und $I$ auf den beiden Zeilen des ersten Stückes und $E$ auf dem zweiten $z u$ erkennen. $P$ nach I hat große Wahrscheinlichkeit für sich, die übrigen Buchstaben sind mehr oder minder zweifelhaft. Es kommt dazu, daß die Natur des Steines verschieden ist von der bekannten, sicher der Papstgruft angehörigen Inschrift des Damasus Hic congesta iacet und daß die Buchstaben kleiner als die dieser sind. Während sich ein Fragment dieser Inschrift in situ gefunden hat, konnte De Rossi über den Platz der anderen, Tempore, nur Vermutungen ohne zwingenden Beweis anstellen. Selbst angenommen also, daß De Rossi das Fragment richtig. bestimmt hat, so läßt sich darum doch nicht mit Sicherheit behaupten, daß die Inschrift zu der Papstgruft gehörte. Die Möglichkeit, daß das Fragment verschleppt ist, muß zugegeben werden. Auch die Krypta der Lucina aber hatte Damasus mit Versen geschmückt, von denen sich Fragmente gefunden haben (I $288 \mathrm{ff}$.). Mit diesen müßte das fragliche Fragment nach der Art des Steines und der Größe der Buchstaben verglichen werden.

Liefie sich nachweisen, daß das Gedicht Tempore von Damasus tatsächlich für die Papstgruft bestimmt war, so wäre damit allerdings die Annahme widerlegt, daß es Stephanus ist, der in ihnen gefeiert wird, da die Angabe der Stephanusakten, daß er in der Krypta der Lucina beigesetzt sei, unbezweifelbar ist. Denn die Bezeichnung coemeterium Lucinae muß auf alter Überlieferung beruhen, da sie nach dem eigenen Zeugnis dieser Akten zu der Zeit, als sie verfaßt wurden, nicht mehr in Gebrauch war. Sie ist uns überhaupt eben nur durch diese bekannt (Roma sott. 1277). Aber damit wäre noch nicht die Beziehung der Inschrift auf Sixtus bewiesen.

Übrigens wurden die Gebeine des Stephanus durch-Papst Paul (757-767) in ein von ihm zu Ehren des Stephanus und Silvester (in honore sancti Stephani, scilicet martyris et pontificis, nec non et beati Silvestri idem pontificis et confessoris Christi) erbautes Kloster versetzt (Liber pontificalis ed. Duchesne I 464), wodurch die Inschrift Tempore, wenn sie zu seinem Grabmal gehörte, gegenstandlos und um so eher der Zerstörung ausgesetzt wurde. 
Eine Schwierigkeit bildet der erste Vers: Tempore quo gladius secuit pia viscera matris, womit anscheinend der Tag des Martyriums umschrieben wird. Die Mutter des Herrn kann mit dieser mater dolorosa nicht gemeint sein. Man könnte an sich an die Mutter der Makkabäer denken, allein deren Fest wurde am 1. August gefeiert. Das Martyrologium Romanum verzeichnet eine andere Mutter, Theodota, die an dem in den Akten als Todestag des Stephanus angegebenen Tage,' dem 2. August, mit drei Söhnen den Tod erlitt. Aber es ist sehr unwahrscheinlich, daß Damasus danach den Todestag des Stephanus bestimmt haben sollte, da Theodota und ihre Söhne erst unter Diocletian das Martyrium erlitten und nicht nachgewiesen werden kann, daß ihr Festtag in Rom schon zu Damasus' Zeit bekannt war. So wird es wohl bei der Erklärung, daß die Mutter Kirche zu verstehen und also nur ganz allgemein die Zeit der Verfolgungen umschrieben sei, sein Bewenden haben müssen.

Lassen sich aber auch nicht alle Schwierigkeiten glatt und sicher lösen, so spricht doch die überwiegende Wahrscheinlichkeit dafür, daß Damasus die Verse Tempore usw. auf Stephanus gedichtet hat.

Das Faktum selbst also, das Cyprian berichtet, daß ein römischer Bischof auf einem Coemeterium hingerichtet worden war, wird, abgesehen von dem Namen, durch die römische Tradition bestätigt. Dabei findet.das, was an der Angabe Cyprians infolge ihrer Kürze unklar ist, insofern eine gewisse Erklärung, als es sich nach den Stephanusakten um die Vollstreckung eines rechtskräftig gefällten Urteils handelt.

Das Martyrium des Sixtus wird auch von Pontius erwähnt (s. XV 292). Er sagt nicht, wie und wo Sixtus den Tod erlitten habe, aber er macht bei dieser Gelegenheit eine sonderbare Bemerkung. Die Lust zur Predigt sei bei Cyprian so groß gewesen, daß er gewünscht habe, das Martyrium möge ihm in der Weise zuteil werden, daß er während der Predigt getötet würde. ${ }^{2}$ An und für sich betrachtet erscheint das als ein ganz unmöglicher Wunsch, da es nach dem gewöhnlichen Lauf der Dinge ausgeschlossen war, daß der Henker ohne weiteres antrat, um den Geistlichen bei der Ausübung seines Amtes niederzuschlagen. Man würde also gar nicht begreifen, wie Cyprian auf solche Gedanken gekommen wäre, wenn ihm nicht ein Fall vorgeschwebt hätte, in

1 in sua decollatus est sede sub die IV Non. Aug.

2 Icb bin auf die Bedeutung dieser Bemerkung erst durch Herrn Professor Dessau in einer Unterhaltung uber das Martyrium des Sixtus aufmerksam gemacht worden, wofür ich ihm auch an dieser Stelle meinen Dank aussprechen möchte. 
welchem durch ganz besondere Umstände ein solches Martyrium zustande gekommen war. Begreiflich wird die Erzählung des Pontius durch das Ereignis, dessen äußere Urnrisse wir in dem Nebel der römischen Tradition erkennen.

Aber die römische Tradition verknüpfte dies Ereignis mit dem Namen des Stephanus, Cyprian mit dem des Sixtus. Die römische Tradition können wir bis ins vierte Jahrhundert zurückverfolgen, dagegen ist Cyprians Nachricht gleichzeitig, und sie ist ihm von Leuten übermittelt, die unmittelbar nach dem Ereignis von seinem Schauplatz zurückgekehrt waren.

$\mathrm{Daß}$ auch Nachrichten dieser Art nicht in jedem Falle zuverlässig sind, lehrt die tägliche Erfahrung. Die Frage ist, ob das oben zitierte Stück der Akten des Sixtus nicht etwa über das vierte Jahrhundert bis auf die Zeit des Martyriums selbst zurückgeführt werden kann, so daß sich in Wahrheit zwei gleichzeitige Zeugnisse gegenüberständen. Der allgemeine legendenhafte Charakter der Akten schließt die Möglichkeit nicht aus, daß einzelne Stücke von der Legende unberührt geblieben wären, und ebenso könnte in den Akten des Stephanus trotz ihres grotesken Wunderglaubens ein historischer Kern stecken.

Allein daß der Name des Stephanus für die Frage, welcher Papst unter Valerian auf einem Coemeterium hingerichtet wurde, gar nicht in Betracht kommen kann und daß dafür tatsächlich nur Sixtus übrig bleibt, läßt sich ganz sicher erweisen. Denn es ist uns ein ausgezeichnetes Mittel für die Kontrolle der Tradition in den Aufzeichnungen des Chronographen vom Jahre 354 erhalten. ${ }^{1}$

Diese Aufzeichnungen enthalten eine Depositio Episcoporum, d. h. eine Angabe des Begräbnistages und -platzes der römischen Bischöfe von Lucius († 255) an, und desgleichen eine Depositio Martyrum. Der Chronograph hat dabei ein älteres Verzeichnis aus dem Jahre 335 benutzt. Die Begräbnistage sind nämlich nicht nach den Jahren, sondern nach den Monaten und Tagen geordnet. Der den Jahren nach letzte ist der des Papstes Silvester ( $\dagger$ 335). Dann folgen, nach den Jahren, nicht nach den Monaten, also noch nicht in die Reihenfolge eingeordnet, Papst Marcus $(\dagger 336)$ und Julius. Für die nach demselben Prinzip angelegte Depositio Martyrum hat man dasselbe Jahr 335 als Abschluß anzunehmen. Denn die enge Zusammengehörigkeit beider

1. Mommsen, Abbandlungen der' Sächs. Gesellsch. der Wiss. phil.-hist. Abt. I 1850 S. 549 ff. Jetzt Monum. Germ. 1892 Chronica minora I. Die Abhandlung, auBer der Ghronik selbst, wiederholt in den kleinen Schriften VII $536 \mathrm{ff}$. 
Listen wird dadurch bewiesen, daß die päpstlichen Märtyrer nicht in der Depositio Episcoporum, sondern in der Depositio Martyrum aufgeführt sind, woraus man übrigens die außerordentliche Hochschätzung der Märtyrer erkennt. Auf diese chronologische Tatsache, die selbstverständlich schon Mommsen gebührend hervorgehoben hat, ist hier besonders $z u$ achten, weil dadurch der Zeitraum zwischen den Aufzeichnungen und dem Pontifikate des Damasus erheblich erveitert wird. Der Chronograph hat ferner ein Verzeichnis der sämtlichen römischen Bischöfe unter Angabe nur ihrer Amtszeit bis auf Urbanus († 231), von da ab unter Hinzufügung historischer Notizen.

Während nun Sixtus ebenso wie der. schon im Jahre 250 gestorbene Fabianus seinen Platz in der Depositio Martyrum gefunden hat, steht Stephanus in der Depositio Episcoporum, wobei übrigens der Tag seiner Depositio mit seinen Akten stimmt, während als Ort das Coemeterium Calisti angegeben ist: IIII Non Ang Steffani in Calisti. Im Jahre 335 war also die Bezeichnung Coemeterium Lucinae bereits außer Gebrauch gekommen und der Name des Calistus auch auf diesen Bezirk ausgedehnt. Auch in dem Verzeichnis sämtlicher Bischöfe ist bei Stephanus einfach das Todesjahr, bei Sixtus die Passion angegeben.

Nach dem Chronographen vom Jahre 354 ist also Stephanus eines natürlichen Todes gestorben.

Aber gegen seine Glaubwürdigkeit sind Bedenken erhoben worden. Außer Stephanus stehen in der Depositio episcoporum auch die Päpste Lucius und Felix, von welchen Lucius $(† 255)$ von Cyprian ausdrücklich als martyr bezeichnet wird, ep. LXVIII 5: servandus est enim antecessormm nostrorum beatornm martyrum Cornelii et Lucii honor gloriosus. Auch Cornelius fehilt in der Depositio martyrum. Bei ihm ist die Kontrolle durch die Depositio episcoporum nicht möglich, weil sein Tod vor dem Jahre 255 erfolgte. Dieses Fehlen beruht nach Mommsen und De Rossi auf einem Versehen der Abschreiber. Der Todestag des Cornelius wurde nach dem ältesten, wohl bis in die Zeit Leos d. Gr. zurückgehenden römischen Sacramentarium an demselben Tage wie der Cyprians, nämlich am 14. September, ${ }^{1}$ und, wie die Ausgrabungen ergeben haben, an demselben Orte, in der Crypta Lucinae, gefeiert. Aber sind wir darum berechtigt, die Notiz des Chronographen: Kal. XVIII Octob. Cypriani Africae, Romae celebratur in Calisti mit De Rossi so zu ergänzen: Kal. XVIII Octob. Corneli in Calisti, Cypriani

1 XVIII Kal. Octob. Natale sanctornem Cornelii et Cypriani. Migne LV 101. 
Africac, Romae celebratur in Calistir' Auch in den biographischen Notizen erwähnt der Chronograph nichts von einem Martyrium des Cornelius. Denn wenn er von ihm sagt: Post hoc Centumcelis expulsi (sic!). Ibi cum gloria dormitionem accepit, so heißt das nichts anderes, als daB Cornelius in der Verbannung gestorben war. Auch Lucius war verbannt worden, aber aus der Verbannung wohlbehalten nach Rom zurückgekehrt: Hic exul fuit et postea nutu dei incolumis ad ecclesiam reversus est. Beide waren also nach dem Chronographen nur confessores, nicht aber martyres im eigentlichen Sinne gewesen. Dagegen verschlägt die Angabe Cyprians nicht viel. Denn Cyprian gebraucht den Ausdruck martyr öfters gleichwertig mit confessor und nennt denselben bald martyr, bald confessor, so einen Jüngling, der wegen seines Bekenntnisses einmal die Verbannung, dann die Folter erlitten hatte (s. S. 581, 12 und 584, 4 Hartel). Das hängt mit seiner Auffassung des Martyriums zusammen. Auch wer die Passion nicht erlitten, aber bewiesen hat, daß er unter Geringschätzung und Aufgebung aller seiner Güter Christus folgt, wird von Christus unter die Märtyrer gerechnet (Ad Fortun. c. 12 S. 345, 10 Hartel). Er wendet das aber auf Lucius selber an in dem Schreiben, in dem er ihm zu der Rückkehr aus seiner Verbannung Glück wünscht (ep. LXI): in confessoribus Christi dilata martyria non meritum confessionis minuunt $(696,3)$. Es sollen nach ihm insbesondere diejenigen Christen, die im Gefängnis sterben, bevor sie das (Martyrium erlitten haben, zu den Märtyrern gerechnet, ihre Tage vermerkt und ebenso wie die der Märtyrer festlich begangen werden (ep. XII 1 und 2). Daher kann es kaum auffallen, wenn er besonders dem Cornelius, der im Exil gestorben war und dessen Tod deswegen auch von dem Chronographen ruhmvoll. genannt wird, den Titel Märtyrer gibt. Er schmückt Cornelius und Lucius aber nicht nur so im Vorbeigehen, wie den Cornelius in dem Briefe an Lucius S. 696, 23, mit diesem Titel, sondern er will dadurch auf ihren Nachfolger Stephanus einen Druck ausüben. Dieser soll dieselbe Praxis hinsichtlich der Lapsi befolgen wie jene, die pleni spiritu Domini et in glorioso martyrio constituti die reuigen Gefallenen wieder in die Kirche aufnahmen. Hier ist unter dem Martyrium offenbar nur das Exil verstanden oder, genauer, das Bekenntnis, das zu dem Exil führte. Das Argument,

i Mommsen wollte, ohne Rücksicht auf das römische Sacramentarium, emendieren: Cypriani Africae. Romae Comeli in Calisti. Abhandlungen der Sächs. Ges. d. Wiss. S. 633 . Nicht aufgenommen in die Chron. min. Aber kl. Schr. VII 553 A. 2: „Cornelius scheint durch Versehen des Schreibers zu fehlen." 
mit dem Cyprian wirken will, ist dies, daß Cornelius und Lucius ihre Entscheidungen als Märtyrer getroffen haben. Durch das Martyrium wurden siè mit dem heiligen Geist erfüllt, aber dieser Umstand hatte doch keine rückwirkende Kraft. Cyprian denkt also an die Entschejdungen, die sie nach ihrer Verurteilung getroffen haben. Das. Martyrium, von dem er spricht, hat weder Lucius noch Cornelius das Leben gekostet und in martyrio constituti heißt tatsächlich nichts anderes als in exilio constituti.

Nun führt De Rossi für den Märtyrertod des Cornelius allerdings noch ein anderes Zeugnis an, die von ihm aufgefundene Inschrift CORNELIVS MARTYR EP aus der Crypta Lucinae. Die Buchstabenform ist nach ihm älter als Damasus, und er nimmt keinen AnstoB, die Inschrift für gleichzeitig mit der Beisetzung zu halten. Wenn irgendeinem, so muß man sicherlich De Rossi darüber ein maßgebendes Urteil zuerkennen. Aber in wissenschaftlichen Fragen darf man sich auch vor der höchsten Autorität nicht ohne weiteres beugen, und da der Annahme eines Märtyrertodes des Cornelius so gewichtige Bedenken entgegenstehen, so wäre es im höchsten Maße wünschenswert, daß das Alter dieser Inschrift mit allen Mitteln noch einmal auf das sorgfältigste geprüft würde. Einstweilen wird man dieses Zeugnis außer Rechnung stellen dürfen.

Inzwischen möchte ich noch eine andere Erwägung gegen das Martyrium des Cornelius ins Feld führen.

Es ist zwar nicht unmöglich, daß Cornelius und Cyprian, wie es in einem der Gebete des alten Sacramentars heißt, zeitlich und örtlich getrennt, doch an demselben Tage den Märtyrertod erlitten hätten. Allein ein eigenes Spiel des Zufalls wäre das doch. Nun haben wir für dieses Datum kein zeitlich bestimmtes älteres Zeugnis als das des alten römischen Sacramentars. Ist es nun nicht eine unerhörte Gewaltmaßregel, wenn wir auf Grund dessen den Text des Chronographen emendieren statt aus dem überlieferten Tatbestand die natürlichen Schlußfolgerungen $\mathrm{zu}$ ziehen? Der Tatbestand ist nach der Überlieferung, daß bis zum Jahre 335 in Rom am 14. September nur der Festtag Cyprians gefeiert wurde, in der Zeit aber zwischen dem Jahre 335 bis zu der Entstehung jenes alten Sacramentars es aufkam, auch den Todestag des Cornelius an demselben Tage zu begehen. Die Erklärung liegt darin, daß beide Feste an demselben Orte, nämlich am Grabe des Cornelius, gefeiert wurden.

Der Tag der Beisetzung des Cornelius war in Vergessenheit geraten, sonst würde die Depositio Episcoporum bei dem Chronographen 
mit Cornelius statt mit seinem Nachfolger Lucius beginnen. Allerdings läßt sich, wenn nicht der Tag der Beisetzung, so doch der Todestag, zwischen welchen beiden Tagen wohl meistens nicht unterschieden wurde, aus den Angaben des Chronographen in dem Verzeichnis sämtlicher Bischöfe berechnen, da wie bei allen andern, so bei Cornelius die Zahl der Tage, Monate und Jahre seines Pontifikats angeführt ist: ann. II m. III d. X. Freilich hat in Wirklichkeit Cornelius nicht so lange regiert, da er im Jahre 251 gewählt wurde und schon im Jahre 252 starb. Vielleicht ist die Zahl gewonnen worden, indem man vom Tode seines Vorgängers am 21. Januar 250 an rechnete, worauf der päpstliche Stuhl längere Zeit unbesetzt geblieben war. Unter dieser Voraussetzung kommt man, da Fabianus am 20. Januar 250 beigesetzt ist, auf den 29. April 252 als Todestag des Cornelius. Da er in der Verbannung gestorben war, so lag zwischen seinem Tode und seiner Beisetzung in der Crypta Lucinae eine kürzere oder längere Zwischenzeit. Aber es ist wenig glaublich, daß das Gedächtnis seines Todestages sich erhalten haben sollte, während das seiner Beisetzung verloren gegangen war. Viel wahrscheinlicher ist es, daß die Zahl der Jahre, Monate und Tage seines Pontifikats auf einer ganz willkürlichen Schätzung beruhen. Dasselbe gilt von der Regierungszeit seiner Nachfolger Lucius und Stephanus. Mit den überlieferten Zahlen ist gar nichts anzufangen. Als Todestag des Lucius ist der 3. März 255 angegeben, als Regierungszeit ann. III $\mathrm{m}$. VIII d. X, als Todesjahr des Stephanus dasselbe Jahr 255, als Tag der Beisetzung der 2. August, als Regierungszeit aber ann. IIII m. II d. XXI. Der Fehler in der Angabe des Jahres durch die Konsuln ist evident, denn die schweren Differenzen dieses Papstes mit Cyprian können sich nicht in der kurzen Spanne vom 3. März bis zum 2. August eines Jahres abgespielt haben. Es ergibt sich aber aus diesen Tatsachen, daß die Depositio Episcoporum bei dem Chronographen von der Angabe der Regierungszeiten in dem Verzeichnis sämtlicher Bischöfe unabhängig ist. Sie wird auf einer im Jahre 255 begonnenen Eintragung des Beisetzungstages des jeweils verstorbenen Papstes beruhen.

War nun der Todestag des Cornelius unbekannt, so war dagegen derjenige Cyprians durch seine Akten gesichert.

Daß man diesen in Rom in der Crypta Lucinae am Grabe des Cornelius feierte, hatte seinen Grund sicherlich darin, daß zwischen beiden $\mathrm{zu}$ ihren Lẹbzeiten ein enges Verhältnis bestand. Es war nur' natürlich, daß man auch das Gedächtnis beider an dem Feste Cyprians miteinander verband und man schließlich, nachdem sich die Vorstellung 
gebildet hatte, daß auch Cornelius den Märtyrertod erlitten habe, die Feier seines Martyriums auf den Tag festsetzte, an dem man seiner inmer gedacht hatte. Nachher schien es dann natürlich so, als sei das Fest des Cornelius von Anfang an das erste gewesen und das Gedächtnis Cyprians hinterher damit verbunden, eine Vorstellung, die sich in den Gebeten an dem Feste deutlich widerspiegelt: Tuanque in sanctis martyribus Cornelio, simul etiam Cy'priano praedicare virtutem usw. (Migne LV 102).

Noch deutlicher läßt sich zeigen, wie der Papst Stephanus in der Vorstellung der Gläubigen allmählich zum Märtyrer wurde.

Nach dem Chronographen wurde am 2. August in Rom kein Gedenktag eines Märtyrers gefeiert. In dem alten Sacramentar aber liest man: IV Non. Aug. Natale Scī Stephani in coemeterio Callisti via Appia. Da an diesem Tage nach der Depositio Episcoporum bei dem Chronographen der Papst Stephanus in Calisti beigesetzt wurde, so denkt man natürlich, daß es sich um den Geburtstag ebendieses Papstes handelt. Nun erfährt man aber keine geringe Uberraschung, wenn man in dem Sacramentar die zahlreichen Gebete für diesen Tag liest: sie beziehen sich sämtlich, nicht auf den Papst Stephanus, sondern auf den Protomartyr, dessen Namen er trägt. In dem siebten Gebet aber stößt man, worauf übrigens schon die beiden Ballerini in der Einleitung zu ihrer Ausgabe $\$ 8$ aufmerksam gemacht haben, auf die Bemerkung: Et ideo nativitatem filii tui merito prae ceteris passionis suae festivitate subsequitur. Zwischen dem 25. Dezember aber und dem 27., dem Feste Johannes' des Evangelisten, sucht man vergebens das des Protomartyrs. Man hat also dieses dem Papst Stephanus zu Ehren auf den Tag seiner Beisetzung verlegt und an seinem Grabe gefeiert. Es ist dann aber auch der weitere Schritt nicht ausgeblieben, daß man die Gebete über den Protomartyr hat fallen lassen und den 2. August geradezu als Geburtstag des Papstes Stephanus gefeiert hat. Diese Stufe der Entwicklung. finden wir in dem Sacramentarium Gregors d. Gr. Hier heißt es: IV Non. Aug. Natalis sancti Stephani episcopi et martyris. Statt der Gebete über den Protomartyr aber findet man das folgende: Deus, qui nos b. Stephani martyris tui atque pontificis annua sollemnitate laetificas, concede, ut cuius natalicia colimus de eiusdem etiam protectione gaudeamus (Migne LXXVIII 128). Das Fest des Protomartyrs aber ist wieder auf den 26. Dezember zurückverlegt: VII Kal. Ian. Nativitas s. Stephani (s. Sp. 33).

So ergibt sich, daß von den Päpsten der 50 er Jahre des 3. Jahrhunderts außer Fabianus nur Sixtus II. den Märtyrertod erlitten hat, 
Cornelius aber, sowic Lucius und Stephanus eines natúrlichen Todes gestorben sind.

Die Akten des Cornelius haben die Bollandisten abzudrucken verschmäbt. Sie geben davon nur den Auszug aus dem Martyrologium Ado's wieder. Das Original findet man bei Schelstrade Antiquitas Ecclesiae $1692 \mathrm{im}$ ersten Bande abgedruckt und besprochen. Von Lucius sind keine Akten erhalten, auch kein Auszug bei Ado, aber aus dem Papstbuch muß man auf die Existenz von solchen im frühen Mittelalter schließen. Mit Recht hat Duchesne die Sätze: qui etiam a Valeriano capite truncatus est III Non. Mart. Hic potestatem dedit onnis ecclesiae Stephano archidiacono suo, dum ad passionem pergeret in dem Leben des Lucius auf eine martyrologische Erzählung zurückgeführt. Es gibt aber dafür noch ein anderes Zeugnis. Die Bollandisten führen einen den Prozeß des Lucius betreffenden Abschnitt aus einem Breviarium Sleswiacense an, in dem es heißt, daß Lucius post multas tribulationes et tormenta ab impiissimo Valeriano praefecto capite truncatur. Das Valerianus praefectus ist gewissermaßen ein Fabrikstempel, den außer den Akten des Sixtus auch die des Abdon und Sennes, ${ }^{1}$ des Laurentius, Hippolytus und der Concordia ${ }^{2}$ tragen, nicht aber die des Stephanus, die diesen Papst richtig in die Zeit des Valerianus und Gallienus setzen.

Von Sixtus hat es keine Akten von der Art, wie sie uns von Cyprian erhalten sind, gegeben, denn die vorhandenen können nicht auf dem Wege der Erweiterung und Umwandlung aus einer so schlichten Erzählung tatsächlicher Ereignisse, wie sie die Akten Cyprians bieten, entstanden sein. Wir dürfen uns durch das Richtige, das in ihrem Schluß enthalten ist, nicht verführen lassen, auf die damit verbundene Angabe, Sixtus sei auf dem Clivus Martis enthauptet worden, irgendwelches Gewicht zu legen. Der Begräbnisplatz und der Beisetzungstag des Sixtus waren Gegenstand allgemeiner Kenntnis, denn dieser wurde ja auf jenem alljährlich gefeiert, dagegen war, wenn sie nicht sogleich aufgeschrieben worden war, die Geschichte seines Leidens der freien Legendenbildung preisgegeben. Der Platz vor dem Tempel des Mars auf dem Clivus Martis kommt in mehreren Märtyrerakten als Richtplatz vor. Das beruht gewiß darauf, daß er tatsächlich als solcher benutzt wurde. Um so weniger ist aber gerade darum im

I S. Acta Sanctorum, Juli t. VII 148.

2 Diese drei nur im Auszug in dem Martyrologium Adonis (Migne I, 123) erhalten.

2.7. 9. 1915. 
einzelnen Falle auf die Angabe, daß das Martyrium auf ihm stattgefunden habe, zu geben.

Man wird vielleicht denken, daß die Akten des Cornelius und Stephanus erst angefertigt seien, nachdem sich der Entwicklungsprozeß, durch den sie im Glauben allmählich und unwillkürlich zu Märtyrern wurden, vollzogen hatte, um so mehr als auch in den Akten des Cornelius, dessen Todestag doch, wie gezeigt, aus der Erinnerung geschwunden war, der Tag des Martyriums verzeichnet ist und auf seine Feier ausdrücklich Bezug genommen wird: (in crypta in cy'miterio Calisti), ubi hodie orationes eorzin florent. .

Allein das ist nicht der Fall. Diese Bemerkung in den Corneliusakten kann ihrer ursprünglichen Form nicht angehört haben, denn die Entstehung aller der genannten Akten ist aus Gründen, die hier nicht ausgeführt werden können, sehr viel früher anzusetzen. Nicht die Feste haben die Märtyrerakten hervorgerufen, sondern vielmehr haben diese zu der Entstehung der Texte mitgewirkt. Gewisse Angaben und Anschauungen sind allen diesen Märtyrerakten gemeinsam, so $\mathrm{da} B$ es wahrscheinlich ist, daß sie gleichzeitig und wohl nicht unabhängig voneinander entstanden sind. Die Regierungszeit des Decius ist weit ausgedehnt, er ist es, der den Krieg in Persien führt, den tatsächlich erst Valerian unternommen hat, und dieser ist unter ihm Praefectus Urbis. In die Regierung des Decius fällt nach ihnen das Episkopat des Cornelius, Lucius und Sixtus, nicht das des Stephanus, das, wie schon gesagt, unter Valerianus und Gallienus gesetzt wird. Offenbar ist die Reihenfolge des Stephanus und Sixtus miteinander verwechselt, und so erklärt es sich, daß auf Stephanus übertragen ist, was Sixtus widerfahren war.

Von der Legende ist, wie ich oben gezeigt $\mathrm{zu}$ haben glaube, schon Damasus abhängig. Das mag auffällig erscheinen, denn Damasus konnte es besser wissen, da ihm das päpstliche Archiv zu Gebote stand. Aber dieses bot seiner Phantasie geringe Nahrung, und warum soll man annehmen, daß er der Legende gegenüber vorsichtiger gewesen sei als Ambrosius?

Die römischen Kirchenbücher sind aber nicht so zuverlässig geblieben, wie sie es um die Mitte des 4. Jahrhunderts waren. Auf das Papstbuch, als dessen Grundlage sich dieselben Aufzeichnungen, die wir bei dem Chronographen vom Jahre 354 lesen, noch deutlich erkennen lassen, haben, wie schon angedeutet, die gefälschten Märtyrerakten Einfluß gewonnen. Von den hier in Betracht kommenden Päpsten ist nur das Leben des Stephanus davon freigeblieben. Wie das

Zeitschr. f. d. neutest. Wiss. 16. Jahrg. 1915. 
Lcben des Lucius, so läßt auch das des Sixtus ihre Spuren erkennen, ganz besonders ist aber das des Cornelius danach erweitert worden. Allein selbst gegenüber der Depositio Martyrum bei dem Chronographen vom Jahre 354 ist Vorsicht geboten. Das geht aus dem Schluß der Akten des Abdos und Sémnes, zweier persischer Könige, hervor. Diese sind schon in der Depositio Martyrum verzeichnet: III Kal. Aug. Abdos et Semnes in Pontiani quod est ad ursum piliatum. Ihre Akten schließen folgendermaßen: Post triduo venit quidam Quirinus Christianus subdiaconus qui manebat ibidem iuxta amphitheatrum et collegit noctu corpora et recondidit in arca plumbea in domo sua ter. tio Kal. Augusti. Postea vero latuerunt corpora sanctorum Abdo et Sennes martymun multos annos usque ad tempora Constantini. De qua re factum est, ut ipsos beatos martyres (locum) revelantes (invenerint) temporibus Constantini iam Christiani. (Tunc〉 levata sunt corpora sancta et translata sunt in coemeterio Pontiani. Die Überführung der Gebeine dieser Märtyrer war also unter Papst Silvester erfolgt, der nach dem Chronographen fuit temporibus Constantini (314-335). Zu den Berichten des Papstbuches über die Fürsorge dieses Papstes für das Andenken der Märtyrer stimmt diese Angabe vortrefflich. Es ist klar, daß die angeblichen Finder schon vorher etwas von den Märtyrern gewußt haben müssen, mit andern Worten, daß die vermeintliche Entdeckung die Existenz der-Akten voraussetzt. Denkbar wäre es an sich, daß sie unmittelbar vorher ad hoc fabriziert worden waren. Aber ihr Inhalt zeigt, daß sie älter sind. Wir brauchen also den guten Glauben der Finder gar nicht $z \mathrm{u}$ bezweifeln. Aber da man diese Mär- tyrer nur aus den Akten kannte, von ihrer Grabstätte aber gar nichts wißte, so konnte natürlich ihre Verehrung erst von dem Tage der Überführung ihres Sarges auf das Coemeterium Pontiani an beginnen. Es ist sehr wahrscheinlich, daß man diesen Tag als den Tag ihrer Depositio bezeichnete und er dann in den Akten als solcher angegeben wurde: Aber möglich ist es immerhin, daß das Datum in den Akten von vornherein stand und vielmehr daher genonmen ist. Jedenfalls aber sind diese beiden Märtyrer tatsächlich erst unter Silvester kreiert worden. Und sie stehen in der Depositio Martyrum des Chronographen! Daraus erkennt man, daß man die Märtyrer dieses Verzeichnisses keineswegs ohne weiteres für historisch halten darf.

Wenn nun in dem Schluß der Sixtusakten kein Zeugnis aus alter. Zeit gesehen werden darf, so scheint er doch in einem Punkt gegenüber Cyprian im Recht zu sein. Nicht vier, sondern nur zwei Diakonen sind mit Sixtus enthauptet worden, wenn wir der Meinung des dama- 
sianischen Zeitalters folgen. Am 6. August ist in Rom sowohl nach dem Chronographen vom Jahre 354 wie auch nach dem alten römischen Sacramentarium an den Märtyrergräbern außer dem Geburtstag des Sixtus nur der des Felicissimus und Agapitus, der beiden Diakonen des Sixtus, gefeiert worden. Die außerdem in dem Papstbuch genannten Namen sind dem Chronographen wie dem Sacramentarium fremd. Es war ein vergebliches Bemühen De Rossis, die überlieferte Lesart des Papstbuches: Qui vero sepultus est in cymiterio Calisti via Appia, nam VI diacones supradicti sepulti sunt in cymiterio Practextati, durch Einschiebung von cum quattuor ex sex supradictis hinter via Appia zu emendieren. Die Zahl VI in dem Papstbuch wird auf Addition der vier ungenannten Diakonen bei Cyprian, zu denen dann später die Namen erfunden wurden, zu den beiden bekannten beruhen.

De Rossi glaubte durch seine Emendation den Vers

Hic comites Xysti portant qui ex hoste tropaca

in der Inschrift des Damasus Hic congesta in der Papstgruft gerecht zu werden. Dieser Vers ist allerdings schwer zu verstehen, aber die Emendation De Rossis würde ihm nicht aufhelfen. Denn Damasus nennt die Gefährten des Sixtus überhaupt, während doch zwei davon auf dem Friedhof des Praetextatus lagen. Die Akten des Sixtus sowie ihre eigenen bringen Laurentianus, Hippolytus und Pontius, deren Beisetzungstage schon von dem Chronographen des Jahres 354 verzeichnet sind, mit Sixtus in Verbindung. Aber auch von ihnen lag nur Pontianus in Calisti. Das Merkwürdigste aber an der Inschrift ist, daß der Papst selbst gar nicht genannt wird, sondern nur seine Gefährten. Und wie kommt es, daß ihnen die Ehre zuteil wird, allen andern vorangestellt $\mathrm{zu}$ werden? Diese und andere Schwierigkeiten, die die Inschrift der Interpretation bietet, vermag ich nicht zu lösen. Aber das scheint mir sicher, daß die Unklarheit der päpstlichen Verse gegen das unzweideutige Zeugnis des Chronographen, wonach Sixtus auf dem Coemeterium des Calistus, seine Gefährten aber auf dem des Praetextatus beigesetzt waren, nicht ausgespielt werden darf. ${ }^{1}$ Denn an noch andere Gefährten des Sixtus außer Felicissimus und Agapitus hat Damasus nicht gedacht. Er würde sonst in der ihnen insbesondere gewidmeten Grabschrift nicht von ihnen fast in den gleichen Aus-

1 Ubrigens sagt De Rossi II 230 selbst, daB die Inschrift des Damasus sich nicht besonders und ausschlieblich auf die Papstgruft, sondern wohl auf das Coemeterium des Callistus überhaupt bezieht. Nicht vielleicht auf die gesamte Gräberanlage an der Via Appia? 
drücken wie von den comites Xysti in der Inschrift Hic congesta gesprochen haben.

Aber che wir die von Cyprian angegebene Zahl kurzerhand verwerfen, müssen wir uns doch die Frage vorlegen, woher denn Damasus wissen konnte, daß Felicissimus und Agapitus Diakone des Sixtus gewesen iwaren. In den Aufzeichnungen des Chronographen steht nichts davon. Da steht nur, daß die beiden an demselben Tage wie Sixtus als Märtyrer beigesetzt waren, von ihrem Stande steht nichts da, noch auch von dem Jahre, in welchem ihr Martyrium stattgefunden hatte. Es ist daher sehr wohl möglich, daß die Meinung des Damasus nur auf einer Kombination beruht, und zwar auf einer Kombination, die nicht ohne Bedenken ist. Denn wie sollte man dazu gekommen sein, die zu gleicher Zeit am gleichen Orte hingerichteten Märtyrer nach dem Tode zu trennen? Wenn aber Damasus, wie sehr wabrscheinlich ist, die Sixtusakten kannte, so hat er seine Kenntnis ebendaher, und dem Zeugnis Cyprians steht also auch in diesem Punkte nicht ein geschichtliches, sondern nur ein apokryphes gegenüber.

Aber Felicissimus und Agapitus wurzeln gar nicht ursprünglich in der Sixtuslegende. Mit dem Gedächtnis des Sixtus ist das des h. Laurentius eng verbunden. Auch Laurentius kommt in den Akten des Sixtus vor, seine Rolle verträgt sich aber so wenig mit der jener beiden, daß unmöglich beide Teile von vornherein miteinander in der Geschichte vorgekommen sein können. Die Laurentiusakten selbst sind nicht mehr vorhanden, wohl aber ein ausführlicher Auszug daraus in dem Martyrologium Adonis. Ein großes Stück davon steht wörtlich in den Sixtusakten und überhaupt nehmen Reden des Laurentius in diesen einen unverhältnismäßig großen Raum ein. Es macht ganz den Eindruck, als seien die Laurentiusepisoden erst später eingeschoben. Nun setzen aber diese durchaus voraus, daß Sixtus allein verhaftet und keinem seiner Diakonen mit ihm zusammen der Prozeß gemacht wurde. Als Laurentius hört, daß Sixtus vom Tempel des Mars, wo man ihn vergebens $\mathrm{zu}$ bewegen gesucht hat, dem Mars $\mathrm{zu}$ opfern, wieder in das Gefängnis geführt wird, redet er ihm auf das eindringlichste $z u$, doch seine Begleitung anzunehmen und ihn mit ihm leiden zu lassen. Die ganze lange Rede dreht sich um den einen Punkt, daß es dem Bischof nicht geziemt, ohne seinen Diakon sich dem Herrn zum Opfer darzubringen: Quo progrederis sine filio, pater? quo, sacerdos sancte, sine diacono properas. Tu nunquam sacrificasti sine ministro nec offerre consueveras. Quid in me ergo displicuit tibi, pater? Num degenerem probasti me? Von Felicissimus und Agapitus ist keine Rede, 
Laurentius wetteifert nicht etwa mit ihnen, er will den Bischof nur nicht allein lassen. So schließt er: Et tu, pater, ostende in filio virtutern tuam et offer quem erudisti, ut securus iudicii tui comitatu nobili pervenias ad coronam. So verbürgt diese Episode jedenfalls eine Form der Legende, nach welcher Sixtus ganz allein das Martyrium erlitt. Diese Form der Legende schimmert aber auch in dem Hauptteil der Akten noch deutlich durch.

Sixtus wird mit den beiden Diakonen vor die Richter geführt: Et praesentatus est nochu Decio et Valeriano cum dicobus diaconibus. Aber in dem dann folgenden Verhör ist von den Diakonen keine Rede. Decius verhandelt nur mit Sixtus, er fordert ihn allein zum Opfer auf und bedroht ihn ganz allein: Sacrifica, nam si non feceris, the eris cxemplum omnium. Decius befiehlt darauf, inn zum Tempel des Mars zu führen, damit er diesem Gott opfere; wenn er sich weigere, ihn einzukerkern (recludite eum in privata Mamurtini). Der beiden Diakonen erinnert sich der Redaktor erst am Ende dieses Abschnittes: Et contempto praecepto Decii (logisches Subjekt ist Sixtus), duxerunt eum in custodiam privatam cum ducobus diaconibus Felicissimo et Agapito.

Auch in der zweiten Gerichtsverhandlung ignoriert Decius wieder die beiden Diakonen vollständig, obwohl wieder ausdrücklich hervorgehoben wird, daß sie zusammen mit Sixtus vorgeführt werden. So wird auch über Sixtus allein von Valerian auf Befehl des Decius das Urteil gesprochen. "Dann ergreifen plötzlich Felicissimus und Agapitus das Wort und warnen die Richter vor den ewigen Strafen. Valerian fährt sie an, nicht wie Angeklagte, die ihres Urteils harren, sondern als wenn sie etwa aus dem Zuschauerraum sich erhoben hätten: Quid diu vivent isti, qui nobis tormenta promittunt? Dann gibt er Befehl, daß man sie zum zweiten Male zum Tempel des Mars führe, damit sie opfern, wenn sie aber nicht wollen, an Ort und Stelle enthauptet werden. Sie werden dann aber nicht allein dahin geführt, sondern auch Sixtus, der sich ja schon geweigert hatte zu opfern und daraufhin soeben verurteilt war! Et ducti foras murum portae Appiae coepit b. Syxtus dicere. Hier ist die fehlerhafte Sprachform, die dem Stil der Akten nicht entspricht, sehr auffällig. Aber auch davon abgesehen, scheint mir die spätere Einarbeitung der beiden evident. Sie verrät sich aber schon gleich bei ihrem ersten Auftreten. Die Erzählung beginnt nämlich so: Nach dem Ende des persischen Krieges kommen Kaiser Decius und der Stadtpräfekt Valerianus nach Rom und geben Befehl, daß Bischof Syxtus mit seinem Klerus sich nachts im Tempel der Tellus stellt. Darauf hält Syxtus eine Ansprache an 
seinen Klerus, in der er ihn an das Beispiel derer, die vor ihnen gelitten haben, und vor allem an das Vorbild des Herrn erinnert. Auf diese Ansprache nehmen die Diakonen Felicissimus und Agapitus das Wort: Et nos sine patre nostro quo ibimus?' Diese Frage ist wie die Faust aufs Auge. Denn die Rede des Sixtus hat keinen andern Sinn als den Klerus aufufordern, mit ihm die Prlifung zu bestehen, während die Frage des Felicissimus und Agapitus voraussetzt, daß er sie allein lassen will. Die Frage aber ist nichts anderes als eine Nachbildung und Umbildung der Frage des Laurentius: Quo progrederis sine filio, pater? Es ist also augenscheinlich, daß Felicissimus und Agapitus erst nachträglich nach dem Vorbilde des Laurentius, ungeschickt genug, in die Legende eingefügt sind.

Felicissimus und Agapitus werden zwei unbekannte Märtyrer gewesen sein, die auf dem Coemeterium des Praetextatus beigesetzt waren. Ihre ursprüngliche Grabschrift ist nicht wieder aufgefunden worden, sondern Fragmente einer, wie es scheint, zu einem Grabtische gehörigen Marmórplatte, auf der ihre Namen in Kursivschrift eingekritzelt sind. Dadurch ist die Stelle sichergestellt, wo man sie seit der Zeit des Damasus verehrte, weiter nichts. So bleibt das einzige Zeugnis für die Zahl der mit Sixtus enthaupteten Diakonen, mit dem wir rechnen können, das Zeugnis Cyprians, von dem wir ausgegangen sind. 